\title{
Obstacles to Progress in MS: A Personal Story
}

\section{David G Haegert}

Department of Pathology, McGill University, Canada

*Corresponding author: David G. Haegert, Department of Pathology, McGill University, Duff Medical Building, Room B22, 3775 rue University, Montreal, QC, Canada H3A 2B4, Tel: 514-398-7192; E-mail:david.haegert@muhc.mcgill.ca

Received date: May 26, 2014, Accepted date: May 27, 2014, Published date: May 30, 2014

Copyright: ( $) 2014$ Haegert DG, et al. This is an open-access article distributed under the terms of the Creative Commons Attribution License, which permits unrestricted use, distribution, and reproduction in any medium, provided the original author and source are credited.

\section{Editorial}

The opportunity to serve on the editorial board of the JMSO is an honour and a privilege. In these early days of the journal I thought it might share my experience that illustrates some obstacles to progress in MS. Most MS research is hypothesis-driven, i.e. is performed within one or more generally accepted frameworks of understanding as to how MS works. Discovery research, including for example transcriptomics and proteomics, asks open-ended questions as to mechanisms, pathways or drug responses in MS without formulating specific hypotheses. In one study, we used a whole genome microarray strategy to compare the gene expression profiles (transcriptomes) of naïve CD4 T-cells isolated from healthy controls (HCs) and from patients with secondary progressive MS (SPMS). Unexpectedly, unbiased hierarchical clustering segregated SPMS patients into two groups, which we termed SP-1 and SP-2. The SP-1 patients upregulated numerous immune genes compared with both HCs and SP-2 patients and SP-1 patients had a significantly shorter RRMS duration than SP-2 patients; the patients had not been preselected based on their RRMS durations. We identified a 5-gene signature that also segregated SP-1 patients from HCs and SP-2. A real-time PCR experiment confirmed significantly increased expression of these genes in SP-1. We also used a systems biology approach that identified interaction of various immune signaling pathways in SP-1 vs. SP-2. We concluded that naive CD4 T-cell immune gene activation identifies MS patients having rapid transition to SPMS. We submitted the work to one journal and then to a second; both journals rejected the manuscript in the absence of confirmatory protein expression or functional data. One comment was that our findings were only speculations without additional evidence. We felt that we faced a bias against microarray-based studies. It would be of interest to know whether others using microarrays have experienced a bias against such studies, and if so, whether they think it is appropriate. We concluded that we had to perform both functional and surface protein expression experiments if we wanted to validate our findings and to publish. T-cell stimulation studies indicated significant functional differences between the naïve CD4 T-cells from SP-1 vs. SP-2 patients. We reduced our 5-gene signature to 3-genes (TLR2, TLR4 and CCR1) and showed increased surface protein expression of these molecules in SP-1 vs. HCs and SP2 in two separate patient-control cohorts. That is, as expected from the microarray studies, we had identified a putative biomarker signature associated with rapid MS progression [1].

Neurology accepted our final manuscript [1]. Before that occurred, we had submitted our microarray, functional and protein expression findings to another journal. One comment focused on the cells that we had studied but not on the findings. The comment was something like the following: the T-cells involved in MS are effector T-cells so that a study of naïve CD4 T-cells is questionable. In other words, our findings were not relevant to MS because we studied the 'wrong' cells. I suggest that this comment represents an example of what Thomas Kuhn termed paradigm paralysis: the inability/unwillingness to consider or accept observations that are outside the current thinking or paradigm [2]. In my opinion, this attitude will not facilitate progress in MS. I am grateful that the reviewers for Neurology had different perspectives. I conclude by suggesting that we need to keep open minds in evaluating MS research, whether it be hypothesis-driven or discovery research, as obviously much remains to be learned.

\section{References}

1. Zastepa E, Fitz-Gerald L, Hallett M, Antel J, Bar-Or A, et al. (2014) Naïve CD4 T-cell activation identifies MS patients having rapid transition to progressive MS. Neurology 82: 681-690.

2. Kuhn TS (1962) The structure of scientific revolutions. University of Chicago Press. 